\title{
Relationship between Lifestyle, Body Mass Index, and Dietary Factors with the Equol Production
}

\author{
Madjid $\mathrm{TH}^{1}$, Anwar $A D^{2}$, Anwar $\mathrm{R}^{3}$, Angganiawati $\mathrm{RD}^{4}$, Mantilidewi $\mathrm{K}^{5}$, Handono $\mathrm{B}^{6}$
}

\begin{abstract}
Aim: This study was aimed to find the relationship between lifestyle factor (smoking and physical activity), body mass index, and dietary component with equol-producing phenotype. Our work can be useful as basic data to modify lifestyle and nutrition to improve the metabolism of equol production, which is needed for women's health.

Materials and methods: Data were collected using interview and semiquantitative food frequency questionnaire. Equol was measured in urine and was collected after a 3-day soy challenge to determine equol-producing phenotypes.

Results: Correlation analysis among lifestyle factors and dietary component with equol-production phenotpe was performed. Data show that equol producers accounted in $60.7 \%$ of the participants. Smoking was significantly correlated to equol-producing phenotype ( $p=0.030$; $r=0.224)$. Carbohydrate and dietary fiber were positively correlated with equol-producing phenotypes $(p=0.011 ; r=0.202)$ and $(p=0.004$; $r=0.218$ ). No significant correlation observed between physical activity, BMI, dietary protein, and fat intake with equol-producing phenotypes $(p=0.677 ; r=0.035)$, although we observed a lower dietary fat intake in equol producer compared to nonequol producer.

Conclusion: These findings suggest that smoking habit, carbohydrate, and dietary fiber significantly influence equol-producing phenotypes. Clinical significance: Our study may be useful as basic data to modify lifestyle and nutrition to improve the metabolism of equol production, which is needed for women's health.

Keywords: Body mass index, Dietary factors, Equol production, Nutrition, Physical activity, Smoking.

Journal of South Asian Federation of Obstetrics and Gynaecology (2020): 10.5005/jp-journals-10006-1749
\end{abstract}

\section{INTRODUCTION}

Equol is a daidzein isoflavones metabolite with the help of intestinal microflora, has a similarity in chemical structure with $17 \beta$-estradiol so that it has an estrogenic activity, and was able to stimulate gene transcription via estrogen receptors. ${ }^{1}$ There are several factors that are known affecting the metabolism of daidzein into equol, including the specific strain of intestinal bacteria, which is able to metabolize daidzein into equol. 2,3 Lifestyle factors, such as, smoking, physical activity, and obesity are also known to influence the composition of the microflora in the gastrointestinal tract, which reduce the diversity of the intestinal microflora and reduce the population of bacteria that are able to convert daidzein into equol. ${ }^{4,5}$ Dietary components like carbohydrates, fats, protein, and fiber also can determine the bacterial population which can grow predominantly in the gastrointestinal tract. Equol production has correlation with the intake of carbohydrates and high fiber. Carbohydrates and fiber that are not digested by the small intestine will undergo fermentation process, which selectively will stimulate the growth and the activity of beneficial intestinal bacteria after entering the colon. Protein and fat intake is known to inhibit the production of equol. They can produce harmful metabolites, which are toxic and carcinogenic, and can inhibit the growth of beneficial bacteria. ${ }^{6}$ There are two phenotypes in the human population, namely, the equol-producing and nonequol-producing. In this study, soy milk was given for three days, for the substrate additional isoflavones in daily diet, as a challenge test to distinguish the two phenotypes of the subjects. Equol levels can be detected in plasma, feces, and urine. However, equol levels in urine are lower than the levels of equol in plasma, and equol detection by using urine is easier and less invasive.

\begin{abstract}
${ }^{1-6}$ Department of Obstetrics and Gynecology, Faculty of Medicine, Padjadjaran University/Dr Hasan Sadikin Hospital, Bandung, West Java, Indonesia

Corresponding Author: Madjid TH, Department of Obstetrics and Gynecology, Faculty of Medicine, Padjadjaran University/Dr Hasan Sadikin Hospital, Bandung, West Java, Indonesia, Phone: +62811210299, e-mail: madjidtita@yahoo.com

How to cite this article: Madjid TH, Anwar AD, Anwar R, et al. Relationship between Lifestyle, Body Mass Index, and Dietary Factors with the Equol Production. J South Asian Feder Obst Gynae 2020;12(1):18-22.
\end{abstract}

Source of support: Nil

Conflict of interest: None

\section{Materials and Methods}

This study was an observational study with cross-sectional method, analyzing the correlation of lifestyle factors (smoking habits and physical activity), body mass index, and dietary components with the equol-producer phenotype. Research samples were obtained from the study subjects who met the inclusion criteria, which were reproductive, perimenopausal, and postmenopausal women who were healthy and were voluntary to be the subject on the study. The exclusion criteria were those subjects who did not follow the whole steps of the study, including those who did not drink soy milk $>1$ day prior to the examination of urinary equol concentration and those who were known based on their history had allergic to soy milk.

The method that was used to differentiate equol-producing and nonequol-producing status on the subjects was by giving the

() The Author(s). 2020 Open Access This article is distributed under the terms of the Creative Commons Attribution 4.0 International License (https://creativecommons. org/licenses/by-nc/4.0/), which permits unrestricted use, distribution, and non-commercial reproduction in any medium, provided you give appropriate credit to the original author(s) and the source, provide a link to the Creative Commons license, and indicate if changes were made. The Creative Commons Public Domain Dedication waiver (http://creativecommons.org/publicdomain/zero/1.0/) applies to the data made available in this article, unless otherwise stated. 
subjects 2 cups $(250 \mathrm{~mL}$ ) daily morning and evening of soy milk in addition to their daily dietary consumption for 3 consecutive days, and then on the third day, 24 hour urine samples were collected for urinary equol concentration examination. Urinary equol concentration is stable at room temperature up to 14 days. Equol examination was conducted by using electrospray ionization mass spectrometry (ESI-MS). Equol-producing groups were determined if the urinary equol concentration was $>20 \mathrm{ng} / \mathrm{mL}$.

Dietary intake of specific compound was obtained by using semiquantitative food frequency questionnaire method so their daily intake of carbohydrates, protein, fat, and fiber could be determined, and then the result was analyzed by using Food processor II version 3 .

\section{Ethics Approval and Consent to Participate}

This study was ethically conducted in accordance with Declaration of Helsinki and was approved by Ethical Clearance Committee Review Board, Dr Hasan Sadikin General Hospital No: LB.04.01/ A05/EC/120/IV/2015.

\section{Results}

This research was conducted from January to June 2015. During the period, 140 subjects met the inclusion criteria. The subjects were reproductive age, perimenopausal, and postmenopausal healthy women. Some were RSHS staffs and group of women from Posbindu Teratai Komplek Bukit Cimindi Raya, Pasirkaliki, Posyandu, and Posbindu in Cileunyi area.

Most of the subjects in both groups were in the perimenopausal age (45-55years). The previous report says that age and menopausal status have no influence on the ability to produce equol. Age only became substantial factor among newborns in the first month of life, compared to infants or adults, due to the absence of intestinal flora in newborn infants (Table 1).

From Table 2, only smoking habit factor had a significant correlation with the equol-producing and nonequol-producing groups. The higher the number of cigarettes consumed, the lower the percentage of equol production. Physical activity did not have a significant correlation between equol-producing and nonequolproducing groups. Body mass index variable tended to give effect that if there is higher BMI for a person, the person's ability to produce equol will be lower, although it was not statistically significant.

Table 3 show higher intakes of carbohydrate and fiber were obtained in the equol-producing group compared to the nonequolproducing group. These variables had positive correlation and statistically significant $(p<0.05)$, although the strength of correlation was weak $(r<0.4)$.

\section{Discussion}

\section{Analysis of Lifestyle Factors (Smoking Habit and Physical Activity) and BMI}

From two lifestyle factors and BMI variables, only smoking habit had significant correlation to equol-producer phenotype. Higher number of cigarettes smoked gave a lower percentage of equol production. This finding was similar to Liu et al. that showed the ratio between smokers in the nonequol-producing group was statistically higher than equol-producing groups. ${ }^{7}$ The effect of smoking in inhibiting the conversion of daidzein into equol was by reducing Bifidobacterium in composition of intestinal microflora. ${ }^{8}$ Besides, smoking has been reported to
Table 1: Characteristics of subjects

\begin{tabular}{|c|c|c|c|}
\hline \multirow[b]{2}{*}{ Variable } & \multicolumn{2}{|c|}{ Equol-producer phenotype } & \multirow[b]{2}{*}{$p$ value } \\
\hline & $\begin{array}{l}\text { Nonequol- } \\
\text { producing } \\
(n=55)\end{array}$ & $\begin{array}{l}\text { Equol- } \\
\text { producing } \\
(n=85)\end{array}$ & \\
\hline \multicolumn{4}{|l|}{ Age (years) } \\
\hline $20-39$ & 10 & 11 & 0.089 \\
\hline $40-59$ & 42 & 62 & \\
\hline$>60$ & 3 & 12 & \\
\hline \multicolumn{4}{|l|}{ Menopause status } \\
\hline Premenopause & 18 & 16 & 0.107 \\
\hline Perimenopause & 23 & 45 & \\
\hline Postmenopause & 14 & 24 & \\
\hline \multicolumn{4}{|l|}{ Education Level } \\
\hline Elementary school & 16 & 42 & 0.074 \\
\hline Junior high school & 2 & 16 & \\
\hline Senior high school & 28 & 14 & \\
\hline Diploma degree & 7 & 8 & \\
\hline Bachelor degree & 2 & 5 & \\
\hline \multicolumn{4}{|l|}{ Occupation } \\
\hline Housewives & 36 & 50 & 0.569 \\
\hline Entrepreneur & 6 & 24 & \\
\hline Civil servant & 9 & 9 & \\
\hline Teacher/lecturer & 4 & 2 & \\
\hline
\end{tabular}

affect mucous production in the colon and affects the immune system in the gastrointestinal mucosa. The differentiation of the environment within the lumen of the intestine allows different types of bacteria to grow, including bacteria that are able to produce equol from daidzein. ${ }^{3}$

In this study, physical activity showed no significant correlation to equol-producer phenotype. This finding was in contradictory to a study conducted by $\mathrm{Wu}$, which showed that isoflavonoid excretion of urine was correlated with regular physical activity. ${ }^{9}$ Another study by Rybak showed that physical activity was significantly correlated with enterolactone concentration in urine which was consistent with a healthy lifestyle. ${ }^{4}$ The mechanism of how physical activity correlated with the ability of converting daidzein into equol was predicted to be the similar to smoking habit, which indicated that lack of physical activity affects the composition of intestinal microflora, which were able to convert daidzein into equol. In addition, lack of physical activity can cause the composition of intestinal microflora to resemble obesity condition.

The lack of significant correlation between physical activity and equol-producer phenotype could be caused by lack of accuracy during data collection. The criteria of physical activity habits on the subject were less strict. Besides, data collection was only gathered through interviews. Observation method would be better to be used for collecting the subjects' physical activity habits. Research regarding correlation of physical activity with equol-producer phenotype between groups of athletes and nonathletes can be conducted to obtain better descriptions.

Body mass index variable also gave no significant correlation. This was similar to Frankenfeld et al., who stated that obesity was not correlated with equol-producer phenotype.Yet in this study, there was a tendency that if a person was more obese, their ability to produce equol was getting lower, even though it was not statistically significant. ${ }^{5}$ One study found that the concentration 
Table 2: Relationship of smoking habit, physical activity, and BMI with equol-producer phenotype

\begin{tabular}{|c|c|c|c|c|}
\hline \multirow[b]{2}{*}{ Variable } & \multicolumn{2}{|c|}{ Equol-producer phenotype } & \multirow[b]{2}{*}{$p$ value } & \multirow[b]{2}{*}{ Phi correlation } \\
\hline & $\begin{array}{l}\text { Nonequol-producing } \\
(n=55)\end{array}$ & $\begin{array}{l}\text { Equol-producing } \\
(n=85)\end{array}$ & & \\
\hline \multicolumn{5}{|l|}{ Smoking habits } \\
\hline Do not smoke & 42 & 75 & $0.030^{*}$ & 0.224 \\
\hline 5-10 cigarettes/day & 7 & 9 & & \\
\hline$>10$ cigarettes/day & 6 & 1 & & \\
\hline \multicolumn{5}{|l|}{ Physical activity } \\
\hline Irregular & 35 & 57 & 0.677 & 0.035 \\
\hline Regular & 20 & 28 & & \\
\hline \multicolumn{5}{|l|}{ BMI } \\
\hline Underweight & 1 & 3 & 0.209 & 0.180 \\
\hline Normal & 30 & 55 & & \\
\hline Overweight & 14 & 21 & & \\
\hline Obesity & 10 & 6 & & \\
\hline
\end{tabular}

*Based on Chi-squared test

Table 3: Relationship between the intake of carbohydrates, protein, fat, and fiber with equol-producer phenotype

\begin{tabular}{|c|c|c|c|c|}
\hline \multirow[b]{2}{*}{ Variable } & \multicolumn{2}{|c|}{ Equol-producer phenotype } & \multirow[b]{2}{*}{$p$ value } & \multirow[b]{2}{*}{$\begin{array}{l}\text { Bi-serial correlation } \\
\text { point }\end{array}$} \\
\hline & $\begin{array}{l}\text { Nonequol-producing } \\
(n=55)\end{array}$ & $\begin{array}{l}\text { Equol-producing } \\
(n=85)\end{array}$ & & \\
\hline \multicolumn{5}{|c|}{ Carbohydrate (g/day) } \\
\hline Mean (SD) & $190.1(72.5)$ & $222.2(78.9)$ & $0.011^{*}$ & 0.202 \\
\hline Median & 180.3 & 219.9 & & \\
\hline Range & $63-363$ & $63-419$ & & \\
\hline \multicolumn{5}{|c|}{ Protein (g/day) } \\
\hline Mean (SD) & $48.7(18.60)$ & $46.9(16.8)$ & 0.736 & -0.051 \\
\hline Median & 45.6 & 46.2 & & \\
\hline Range & $15-99$ & $15-112$ & & \\
\hline \multicolumn{5}{|l|}{ Fat (g/day) } \\
\hline Mean (SD) & $66.41(34.86)$ & $55.8(30.1)$ & 0.109 & -0.161 \\
\hline Median & 57.9 & 52.5 & & \\
\hline Range & $10-157$ & $5-137$ & & \\
\hline \multicolumn{5}{|l|}{ Fiber (g/day) } \\
\hline Mean (SD) & $9.76(4.06)$ & $11.4(3.35)$ & $0.004^{*}$ & 0.218 \\
\hline Median & 9.4 & 11.0 & & \\
\hline Range & $2.8-21.4$ & $3-18.7$ & & \\
\hline
\end{tabular}

*Statistically significant $(p<0.05)$

of several isoflavones, such as, enterodiol and enterolactone, was weakly and inversely correlated with body mass index. ${ }^{9}$

The mechanism of how obesity affects the metabolism ability of daidzein into equol was predicted relative to the change of intestinal microflora composition in obese individuals. Previous research described that human with obesity had more Firmicutes species, which is a type of bacteria that is known to be able to metabolize daidzein into equol. ${ }^{10}$

\section{Effect of Dietary Component Analysis (Intake of Carbohydrates, Protein, Fat, and Fiber)}

\section{Carbohydrate Intake}

Another factor that can affect the production of equol is the differences of dietary intake. Many studies reported correlation of equol-producing group with components of the dietary intake including carbohydrates, proteins, fats, PUFAs, dairy products, lactose, green tea, seaweed, and soy intake, but definitive conclusions were not yet ascertain. Long-term of soy consumption was also not a factor that affects the formation of equol, but the intake of food containing daidzein substrates, such as, soy, and the presence of bacteria in the intestine are essential.

In this study, the analysis of nutrient intake was taken by using the interview reports with semiquantitative food frequency questionnaire method; the analysis was divided based on equolproducing group and nonequol-producing group. The data obtained showed that there was a positive correlation between the intake of carbohydrates and fiber with equol-producer phenotype. The data signified that there was a higher carbohydrate intake on equol-producing group and it was statistically significant although there was a weak strength of correlation. 
High dietary intake of carbohydrates was known to stimulate the production of equol in individuals who had equol-producing bacteria species in their intestine. Previous research by Setchell showed a differentiation of carbohydrate intake between the equolproducing and nonequol-producing groups. ${ }^{6}$ Observational study using fecal flora culture in an environment of high polysaccharides stimulated the fermentation of bacteria that can change daidzein into equol faster. Unfortunately, in the conditions that are similar to the low carbohydrate intake, equol was not formed. Yuan et al. stated that equol production was stimulated by the amount of hydrogen gas that might act as an electron donor in the biotransformation reaction of daidzein into equol. This finding indicated that hydrogen has an important role in the production mechanism of equol. ${ }^{11}$ Slavin et al. showed that inulin, oligofructose, lactulose, resistant starch or other fiber types, and prebiotic carbohydrates were able to stimulate the growth of Bifidobacterium, which was a type of bacteria that is capable to convert daidzein into equol. ${ }^{12}$

\section{Fiber Intake}

The analysis showed that fiber intake has a significant correlation with equol-producer phenotype. This finding was similar to previous study by Lampe which stated that women who excreted equol were consuming more fiber and vegetation protein than those who did not excrete equol. This finding assumed that on those women, fibers or other high-fiber components increased the growth and/or the activity of bacteria that play a role in the production of equol in the intestine. In addition, the equolproducing group who were vegetarian produced 4:25 times higher of S-equol compared to nonvegetarian. ${ }^{12}$

Fiber is composed by carbohydrates, which is resistant to digestion and absorption process in the small intestine. Dietary fiber forms are resistant starch, nonstarch polysaccharides (cellulose, hemicellulose, pectin, and gums), oligosaccharides, lignin, and other substances. Fiber can undergo fermentation in the colon. The mechanism of action of fiber as a prebiotic undergoes fermentation in the colon and alters the composition of intestinal microflora. The process of fermentation in the colon stimulates the growth of beneficial bacteria colonies such as Bifidobacterium type and inhibits pathogen bacteria. ${ }^{13}$ The bacteria has the same characteristic with the bacteria which is capable to convert daidzein into equol.

\section{Protein Intake}

There was no significant correlation between protein and fat components with the equol-producer phenotype found in this study. Previous research conducted by Rowland mentioned that the consumption of meat and high fat could inhibit the intestinal flora, which was needed for the production of equol. High-protein diet also increased the proteolytic fermentation process which can produce useful substances, but the decay process can also produce substances that are toxic to the body. Those toxic substances were ammonia, amines, phenols, and sulfides, and it also has a role in colorectal cancer. Previous researches described that a high-protein diet could increase Bacteroides type of bacteria..$^{14,15}$

Earlier said that the intake of protein did not affect significantly to the ability of converting daidzein into equol and can be caused by a high intake of protein cannot increase the specific bacterial strain, which was able to convert daidzein into equol. In this case, further research was needed to detect the specific strain which was affected by the presence of high levels of protein in the digestive tract. In addition, the effect of protein intake on equol-producer phenotype in our study was affected by its confounding factors, including other macronutrient components. Further research should be done to minimize the effect of other macronutrient components in the subject's intake.

\section{Fat Intake}

In this study, we used the total fat intake as a dietary intake of fat. The analysis of fat intake showed that there was no significant correlation between fat intakes and equol-producer phenotype. Data showed that fat intake on equol-producing group was lower than the nonequol-producing group, although it was not statistically significant. Rowland's research conducted on healthy adults had similar findings, which stated that a good equol-producing group consumed less fat than the nonequol-producing group. ${ }^{16}$ High-fat diet, approximately $49.5 \%$, may alter the composition of intestinal microflora, eliminating Bifidobacterium spp., Clostridium Eubacterium rectale-coccoides, and Bacteroides. The reduction of beneficial bacteria strain will also affect the person's ability to convert daidzein into equol. Furthermore, saturated fat diet can increase pro-inflammatory intestinal microbes by stimulating the formation of taurine-conjugated bile that enhances the growth of pathogenic bacteria. ${ }^{17,18}$

\section{Conclusion}

Smoking habit and the intake of carbohydrates and fiber in dietary consumption had an effect on the equol-producer phenotype.

\section{Clinical Significance}

Our study may be useful as basic data to modify lifestyle and nutrition to improve the metabolism of equol production, which is needed for women's health.

\section{Acknowledgment}

This work was supported by Posbindu Teratai.

\section{References}

1. Setchell KDR, Brown NM, Lydeking-Olsen E. The clinical Importance of the metabolite equol: a clue to the effectiveness of soy and its isoflavones. J Nutr 2002;132(12):3577-3584. DOI: 10.1093/ jn/132.12.3577.

2. Atkinson $\mathrm{C}$, Berman $\mathrm{S}$, Humbert $\mathrm{O}$, et al. In vitro incubation of human feces with daidzein and antibiotics suggests interindividual differences in the bacteria responsible for equol production. J Nutr 2004;134(3):596-599. DOI: 10.1093/jn/134.3.596.

3. Atkinson C, Frankenfeld CL, Lampe JW. Gut bacterial metabolism of the soy isoflavone daidzein: exploring the relevance to human health. Exp Biol Med (Maywood) 2005;230(3):155-170. DOI: $10.1177 / 153537020523000302$.

4. Rybak ME, Sternberg MR, Pfeiffer CM. Sociodemographic and lifestyle variables are compound- and class-specific correlates of urine phytoestrogen concentrations in the U.S. population. J Nutr 2013;143(6):986S-994S. DOI: 10.3945/jn.112.172981.

5. Frankenfeld $\mathrm{CL}$, Atkinson $\mathrm{C}$, Wähälä $\mathrm{K}$, et al. Obesity prevalence in relation to gut microbial environments capable of producing equol or O-desmethylangolensin from the isoflavone daidzein. Eur J Clin Nutr 2014;68(4):526-530. DOI: 10.1038/ejcn.2014.23.

6. Setchell KDR, Brown NM, Summer S, et al. Dietary factors influence production of the soy isoflavone metabolite $\mathrm{S}-(-)$ equol in healthy adults. J Nutr 2013;143(12):1950-1958. DOI: 10.3945/jn.113. 179564. 
7. Liu W, Tanabe M, Harada KH, et al. Levels of urinary isoflavones and lignan polyphenols in Japanese women. Environ Health Prev Med 2013;18(5):394-400. DOI: 10.1007/s12199-013-0338-6.

8. Allais L, Kerckhof FM, Verschuere $S$, et al. Chronic cigarette smoke exposure induces microbial and inflammatory shifts and mucin changes in the murine gut. Environ Microbiol 2015;18(5):1352-1363. DOI: 10.1111/1462-2920.12934.

9. Wu X, Cai H, Gao YT, et al. Correlations of urinary phytoestrogen excretion with lifestyle factors and dietary intakes among middleaged and elderly Chinese women. Int J Mol Epidemiol Genet 2012;3(1):18-29.

10. Kotzampassi K, Giamarellos-Bourboulis EJ, Stavrou G. Obesity as a consequence of gut bacteria and diet interactions. ISRN Obes 2014;2014:651895. DOI: 10.1155/2014/651895.

11. Yuan JP, Wang JH, Liu X. Metabolism of dietary soy isoflavones to equol by human intestinal microflora-implications for health. Mol Nutr Food Res 2007;51(7):765-781. DOI: 10.1002/mnfr.200600262.

12. Slavin J. Fiber and prebiotics: mechanisms and health benefits. Nutrients 2013;5(4):1417-1435. DOI: 10.3390/nu5041417.

13. Parnell JA, Reimer RA. Prebiotic fiber modulation of the gut microbiota improves risk factors for obesity and the metabolic syndrome. Gut Microbes 2012;3(1):29-34. DOI: 10.4161/gmic 19246.

14. Lopez-Legarrea $P$, Fuller NR, Zulet $M A$, et al. The influence of mediterranean, carbohydrate and high protein diets on gut microbiota composition in the treatment of obesity and associated inflammatory state. Asia Pacific J Clin Nutr 2014;23(3):360-368.

15. Russell WR, Gratz SW, Duncan SH, et al. High-protein, reducedcarbohydrate weight-loss diets promote metabolite profiles likely to be detrimental to colonic health. Am J Clin Nutr 2011;93(5):1062-1072. DOI: 10.3945/ajcn.110.002188.

16. Rowland IR, Wiseman $H$, Sanders $T A$, et al. Interindividual variation in metabolism of soy isoflavones and lignans: influence of habitual diet on equol production by the gut microflora. Nutr Cancer 2000;36(1):27-32. DOI: 10.1207/S15327914NC3601_5.

17. Moreira APB, Texeira TF, Ferreira AB, et al. Influence of a high-fat diet on gut microbiota, intestinal permeability and metabolic endotoxaemia. Br J Nutr 2012;108(5):801-809. DOI: 10.1017/S0007114512001213.

18. Hildebrandt MA, Hoffmann C, Sherrill-Mix SA, et al. High fat diet determines the composition of the murine gut microbiome independently of obesity. Gastroenterolgy 2009;137(5):1716-24.e1-2. DOI: 10.1053/j.gastro.2009.08.042. 